\title{
Multiple founder effects in spinal and bulbar muscular atrophy (SBMA, Kennedy disease) around the world
}

\author{
Annastiina Lund*,1, Bjarne Udd ${ }^{2,3}$, Vesa Juvonen ${ }^{1}$, Peter M Andersen ${ }^{4}$, Kristina Cederquist ${ }^{5}$, \\ Mark Davis ${ }^{6}$, Cinzia Gellera ${ }^{7}$, Christina Kölmel $^{8}$, Lars-Olof Ronnevi $^{9}$, \\ Anne-Dorte Sperfeld ${ }^{10}$, Sven-Asger Sörensen ${ }^{11}$, Lisbeth Tranebjaerg ${ }^{12}$, \\ Lionel Van Maldergem ${ }^{13}$, Mitsunori Watanabe ${ }^{14}$, Markus Weber ${ }^{15}$, Leone Yeung ${ }^{16}$ and \\ Marja-Liisa Savontaus ${ }^{1,17}$
}

${ }^{1}$ Department of Medical Genetics, University of Turku, Finland; ${ }^{2}$ Neurological Department, Vasa Central Hospital, Vasa, Finland; ${ }^{3}$ Department of Neurology, University of Tampere, Finland; ${ }^{4}$ Department of Neurology, University of Umea, Sweden; ${ }^{5}$ Department of Medical Genetics, University of Umea , Sweden; ${ }^{6}$ Department of Neuropathology, Royal Perth Hospital, Australia; ${ }^{7}$ Department of Biochemistry and Genetics, Istituto Nazionale Neurologico, Milan, Italy; ${ }^{8}$ Department of Neurology, University Hospital Homburg, Germany; ${ }^{9}$ Department of Neurology, Karolinska Sjukhuset, Stockholm, Sweden; ${ }^{10}$ Exp. Neurologie, Ulm, Germany; ${ }^{11}$ Department of Medical Genetics, University of Copenhagen, Denmark; ${ }^{12}$ Department of Medical Genetics, University Hospital of Tromsoe, Norway; ${ }^{13}$ Centre de Génétique Humaine, Institut de Pathologie et de Génétique, Loverval, Belgium; ${ }^{14}$ Department of Neurology, Gunma University School of Medicine, Gunma, Japan; ${ }^{15}$ Neuromuscular Diseases Unit, Vancouver Hospital and Health Sciences Centre, Canada; ${ }^{16}$ Molecular Medicine Laboratory, University of Sydney, Australia; ${ }^{17}$ Department of Biology, University of Turku, Finland

SBMA (spinal and bulbar muscular atrophy), also called Kennedy disease, is an X-chromosomal recessive adult-onset neurodegenerative disorder caused by death of the spinal and bulbar motor neurones and dorsal root ganglia. Patients may also show signs of partial androgen insensitivity. SBMA is caused by a CAG repeat expansion in the first exon of the androgen receptor (AR) gene on the X-chromosome. Our previous study suggested that all the Nordic patients with SBMA originated from an ancient Nordic founder mutation, but the new intragenic SNP marker ARd12 revealed that the Danish patients derive their disease chromosome from another ancestor. In search of relationships between patients from different countries, we haplotyped altogether 123 SBMA families from different parts of the world for two intragenic markers and 16 microsatellites spanning $25 \mathrm{cM}$ around the AR gene. The fact that different SBMA founder haplotypes were found in patients from around the world implies that the CAG repeat expansion mutation has not been a unique event. No expansion-prone haplotype could be detected. Trinucleotide diseases often show correlation between the repeat length and the severity and earlier onset of the disease. The longer the repeat, the more severe the symptoms are and the onset of the disease is earlier. A negative correlation between the CAG repeat length and the age of onset was found in the 95 SBMA patients with defined ages at onset. European Journal of Human Genetics (2001) 9, 431-436.

Keywords: SBMA; founder effect; Kennedy disease; CAG repeat disorder; trinucleotide repeat

*Correspondence: A Lund, Department of Medical Genetics, University of Turku, Kiinamyllynkatu 10, FIN-20520 Turku, Finland.

Tel: +358 2333 7456; Fax: +358 2333 7300; E-mail: anniemi@utu.fi Received 2 January 2001; revised 22 March 2001; accepted 22 March 2001
Introduction

SBMA (Spinal and Bulbar Muscular atrophy), also called Kennedy disease (MIM 313200), is an X-chromosomal recessive neurodegenerative adult-onset disorder in men. ${ }^{1}$ SBMA is a panethnic disease with prevalence of less than $1 / 40000$ men. ${ }^{2}$ Patients show slowly progressive spinal and 
bulbar muscular atrophy with fasciculations and generalised muscle weakness, frequently sensory symptoms, facial and perioral contraction fasciculation and dysarthria, dysphonia and dysphagia. These symptoms are caused by progressive death of the spinal and bulbar motor neurones and dorsal root ganglia. ${ }^{3}$ Patients frequently show signs of partial androgen insensitivity with gynecomastia, impotence, testicular atrophy and reduced fertility. ${ }^{4}$

SBMA is caused by a CAG repeat expansion in the first exon of the androgen receptor $(A R)$ gene. ${ }^{5}$ Patients have about twice as many CAG repeats as unaffected males, 38-72 repeats, ${ }^{6,7}$ compared with $10-36$ for unaffected males. ${ }^{8}$ SBMA was the first disease in which a CAG repeat expansion was found. At least eight such diseases are known: Huntington disease, DRPLA (dentatorubral-pallidolyusian atrophy) and spinocerebellar ataxias SCA1, SCA2, SCA3, SCA6 and SCA7. ${ }^{9}$

Trinucleotide diseases often show correlation between the repeat length and severity and earlier onset of the disease. The longer the repeat, the more severe the symptoms are and the earlier the onset of the disease. ${ }^{10,11}$

Most Finnish SBMA patients come from the district of Vaasa in Western Finland. ${ }^{3}$ We have shown that all the Finnish families share identical alleles for at least five nearby markers around the AR gene. Because the majority of our patients were Swedish speaking, we extended the study to patients from the linguistically related neighbouring Scandinavian countries. The Finnish, Swedish, Norwegian and Danish SBMA families shared the same intragenic GGC allele, as well as similarities in allele distribution, which suggests a common origin for the Scandinavian SBMA mutation. There also seems to be a specific, more extended sub-founder haplotype in each country. ${ }^{12}$

Both in myotonic dystrophy (DM) ${ }^{13,14}$ and Friedreich's ataxia $^{15}$ a predisposing haplotype associated with long normal repeats explains the founder effects found in these diseases. This prompted us to include in the study SBMA patients from around the world to test whether a common founder haplotype is shared by patients from different countries.

\section{Material and methods}

The patient material consisted of 123 SBMA families with a total of 151 patients and 10 carriers from 10 different countries: Finland, Sweden, Norway, Denmark, Germany, Belgium, Italy, Japan, Australia and Canada (Table 1). The patient material was collected through the neurologists from these countries. Blood samples were drawn after informed consent was obtained. The German patients were from two different hospital districts, as were the Australian patients. Families GI-GV came from Ulm, GVI-GXIII came from Saar, AI-AIX were from Sydney and AX-AXV from the Perth district. The mothers of the Canadian patients were from the following ethnic backgrounds: CI Saskatchewan Indian; CII
Cree Indian; CIII Coast Salish Indian; CIV, CVI and CVIII English; CV Irish; CVII Chinese; CIX Scottish. Families FIFXIII, SI-SX, DI-DXII, NI and NII were previously haplotyped for the 17 microsatellite markers, ${ }^{12}$ and in this study only the intragenic SNP marker was determined for these families.

The age of onset was known for 95 SBMA patients (one Australian, one Belgian, one Norwegian, 18 Finnish, seven Swedish, 13 German, 30 Italian, nine Canadian and 15 Japanese patients), the first symptom being any of the characteristic SBMA symptoms. The dependence between the CAG repeat length and the age at onset was examined using Spearman rank order correlation. The correlation was calculated for all the nationalities together and separately inside the countries.

The SBMA patients were haplotyped for the intragenic SNP marker ARd12 (Whitehead cSNP Data) (http://waldo.wi.mit. edu/cvar_snps/) that changes a cutting site of StuI restriction enzyme, the intragenic GGC repeat and 16 other microsatellite markers (DXS8062, DXS8032, ALAS2, DXS1213, DXS1194, DXS1111, DXS135, DXS1125, DXS981, DXS1689, DXS339, DXS106, DXS1216, DXS1275, DXS453, DXS1690) spanning a $25.2 \mathrm{cM}$ region around the androgen receptor gene. The locations and distances ${ }^{12}$ of the markers were obtained from The Genome Database (GDB) (http:// gdbwww.gdb.org/). The microsatellites were amplified by a standard PCR method. ${ }^{12}$

\section{Results}

The haplotypes of the patients are illustrated in Figure 1. All the Finnish, Swedish and Norwegian patients carried the same kind of haplotype with absent intragenic ARd12 StuI site (minus-allele), while the patients from other countries harboured the StuI site (plus-allele) (Figure 1). All of the German SBMA families carried at least a part of either of the two DXS8062-DXS1690 haplotypes spanning $25.2 \mathrm{cM}, 4-3-5-$ 2-9-(mut)-+-17-4-4-6-6- -15-7-10-3-7-5-3 and 4-7-3-2-3(mut)-+-17-3-4-5-4- -4-2-8-1-5-2-13. The only Belgian patient harboured a unique DXS8062-DXS1690 haplotype not found in any other patients. The Italian patients carried at least three different founder haplotypes with either 17,18 or 13 GGC repeats. In the Japanese families at least two founder haplotypes were found, one with a maximum length only between the loci DXS8062-DXS1125 (14.4 cM). Twelve of the 15 Australian families had at least parts of the two DXS8062DXS1690 haplotypes 4-2-2-6-7-(mut)-+-17-2-6-4-7+-5-7-9-24-2-3 and 4-2-1-2-3-(mut)-+-18-3-4-4-6- -5-7-3-2-5-3-8. No common haplotype was detected among the Canadian patients, but two of them (CIV1 and CVIII1) carried haplotypes similar to the two Australian founder haplotypes, DXS8032-AR GGC (8.5 cM) and DXS8062-DXS1690 $(\geqslant 25.2 \mathrm{cM})$, respectively (Figure 1$)$.

The 123 SBMA families from around the world displayed a CAG repeat number ranging from 35 to 57 with the 


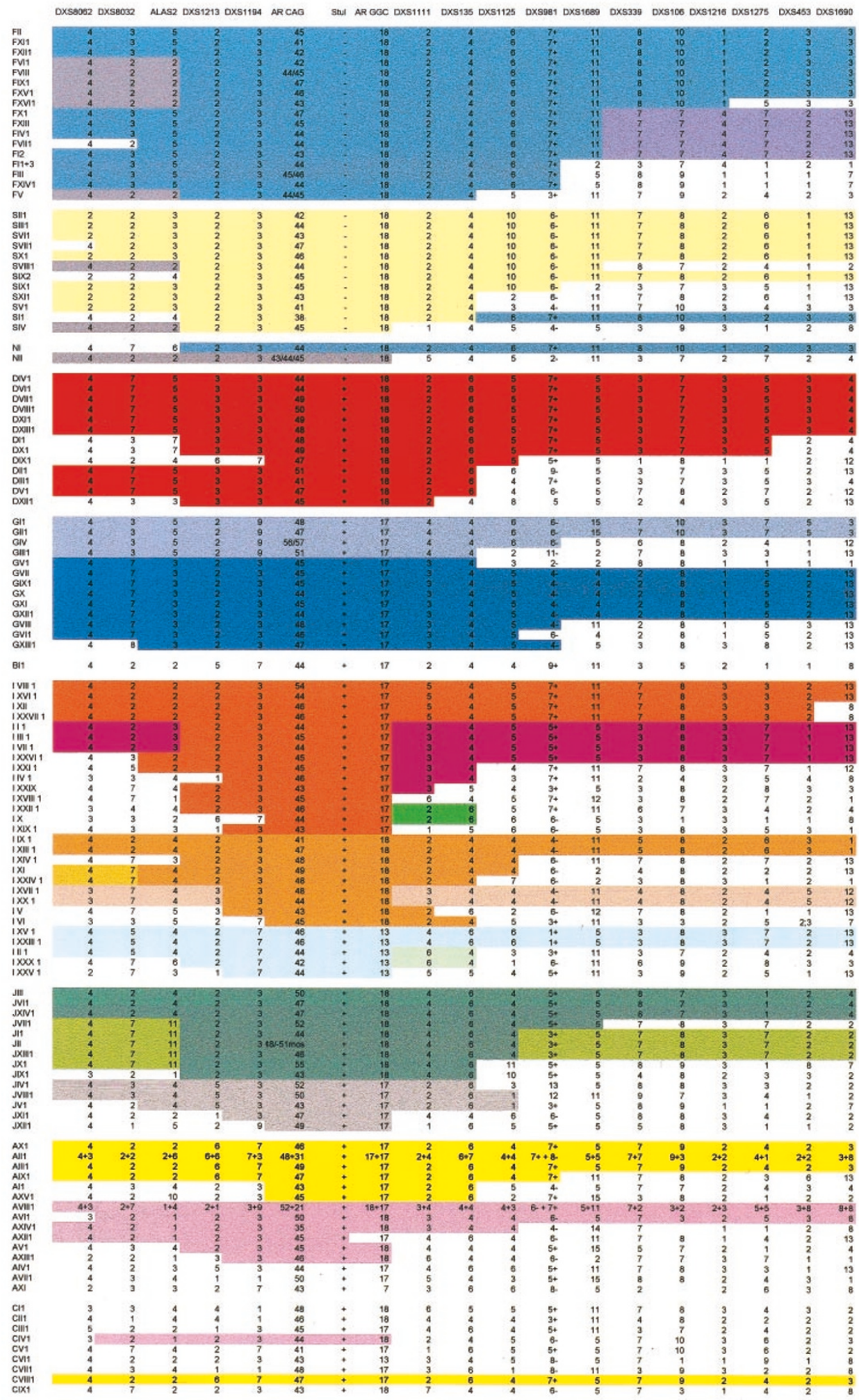

Figure 1 The haplotypes of the Finnish, Swedish, Danish, Norwegian, German, Belgian, Italian, Japanese, Australian and Canadian SBMA families. Different founder haplotypes are shaded with different colours. The number after the family symbol stands for the individual in the family. When there is no number after the symbol, all the family members examined shared the same haplotype. Different CAG numbers in families FIII, FV and NII are shown by multiple CAG numbers. 
Table 1 Number of SBMA families, patients and carriers from different countries

\begin{tabular}{llcc}
\hline Country & Families & Patients & Carriers \\
\hline Finland & $16(\mathrm{FI}-\mathrm{FXVI})$ & 23 & 4 \\
Sweden & $11(\mathrm{SI}-\mathrm{SXI})$ & 15 & \\
Norway & 2 (NI and NII) & 3 & 3 \\
Denmark & 13 (DI-DXIII) & 13 & \\
Germany & $13(\mathrm{GI}-\mathrm{GXIII})$ & 19 & 1 \\
Belgium & 1 (BI) & 1 & \\
Italy & $29(\mathrm{IIIIXXX)}$ & 37 & \\
Japan & $14(\mathrm{II}-\mathrm{JIIV})$ & 16 & \\
Australia & $13(\mathrm{Al}-\mathrm{AXV})$ & 15 & 2 \\
Canada & 9 (Cl-CIX) & 9 & \\
Total & 123 & 151 & 10 \\
\hline
\end{tabular}

Table 2 The CAG repeat ranges and mean CAG numbers of the SBMA patients

\begin{tabular}{lll}
\hline Country & CAG repeat range & Mean CAG no. \pm st dev \\
\hline Finland & $41-47$ & $44.3 \pm 1.6$ \\
Sweden & $38-47$ & $43.5 \pm 2.5$ \\
Norway & $43-45$ & \\
Denmark & $41-51$ & $47.0 \pm 2.8$ \\
Germany & $44-57$ & $47.7 \pm 4.2$ \\
Belgium & 44 & \\
Italy & $41-54$ & $45.3 \pm 2.4$ \\
Japan & $43-55$ & $48.1 \pm 3.5$ \\
Australia & $35-52$ & $45.9 \pm 4$ \\
Canada & $41-48$ & $45.0 \pm 2.4$ \\
For all & $35-57$ & $45.7 \pm 3.2$ \\
\hline
\end{tabular}

mean of $45.7 \pm 3.2$. The $\mathrm{CAG}$ repeat number ranges and mean CAG numbers of each country are shown in Table 2 . In one family, JII, mosaicism was present in one of the two family members. Both JII family members carried 48 CAG repeats, but in one of them longer, up to $51 \mathrm{CAG}$ repeats were also seen (Figure 1).

Among the 95 SBMA patients with defined ages at onset a negative correlation between the CAG repeat length and the age of onset was found $(R=-0.30, P=0.0027)$ (Figure 2$)$. When the different countries were studied separately, a negative correlation was present only among the Japanese patients $(R=-0.60, P=0.018)$. Such a correlation was not found among the Finnish $(R=-0.04, P=0.87)$, Swedish $(R=0.05$, $P=0.91)$, German $(R=-0.16, P=0.60)$, Italian $(R=-0.17$, $P=0.36)$ or Canadian $(R=-0.07, P=0.83)$ patients.

\section{Discussion}

Before discovering the new intragenic SNP marker ARd12 (StuI site), it was concluded that all the Nordic patients with SBMA originated from an ancient Scandinavian founder haplotype. ${ }^{12}$ However, the fact that the Danish patients carry the StuI site, which is absent in the Finnish, Swedish and Norwegian patients, revealed that at least the Danish patients derive their disease chromosome from another ancestor. The similar Finnish, Swedish and Norwegian SBMA haplotypes still indicate a common genetic background for SBMA patients in these three countries. Even though the difference between the Danish and the other Scandinavian haplotypes

\section{Age at onset vs. CAG repeat number}

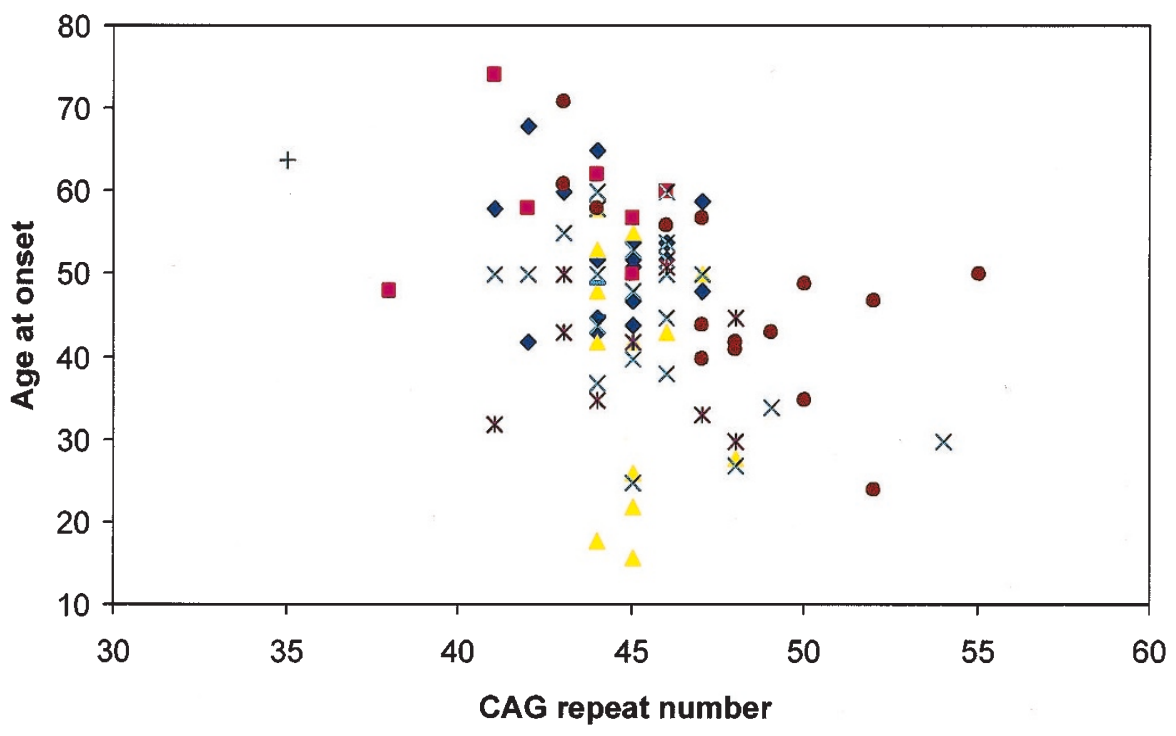

- Finnish patients

- Swedish patients

German patients

$\times$ Italian patients

* Canadian patients

- Japanese patients

+ Australian patient

- Belgian patient

- Norwegian patient

Figure 2 The negative correlation between the CAG repeat number and age at onset of SBMA. 
at ARd12 might be due to gene conversion between the + and - alleles, it cannot be confirmed.

While the Danish SBMA haplotypes resembled German haplotypes in harbouring the StuI site, all the Danish patients carried 18 GGC repeats in the other intragenic marker, contrary to 17 found in the German samples. This indicates that in spite of geographical proximity, the Danish and German SBMA patients most probably originate from different founder mutations. Although microsatellites are prone to mutate occasionally with a rate of $\sim 2 \times 10^{-3}-10^{-4}$ per locus per generation, ${ }^{16}$ it is unlikely that the SBMA mutation of these two countries was of the same origin, because of the dissimilarity of the extended haplotypes.

All the German patients from Saar (GVI-GXIII) harboured a similar haplotype, and one of the patients from Ulm (GV1) carried a haplotype common to the patients from Saar. That is not very surprising, since these places are not far apart. The 'Saar' and 'Ulm' haplotypes harbour similar alleles in the intragenic markers, + allele for StuI and 17 GGC repeats, but the flanking markers DXS1194 and DXS1111, as well as most of the other microsatellite markers studied, are different in the patients from these two areas. Since all the German patients carry similar intragenic alleles, it is possible that the patients from Saar and Ulm have inherited a common ancestral mutation. The Belgian patient had a unique haplotype, also harbouring the intragenic StuI site and 17 GGC repeats. As this is the only sample from Belgium, the origin of this mutation cannot be estimated. The mutation, although unlikely, might have a common origin with the German patients, considering the geographical proximity. However, these intragenic alleles are very common, as StuI site is carried by about $85 \%$ (Whitehead cSNP Data) and 17 GGC repeats is found in about half of the control population. ${ }^{12,17}$ Therefore, the SBMA mutation is likely to have occurred more than once in an AR gene with the StuI site and 17 GGC repeats.

The Italian patients showed three entirely different founder haplotypes with either 13, 17 or 18 intragenic GGC repeats, and thus the SBMA mutation in Italy has probably at least three different origins. The Japanese patients had two distinct haplotypes with 17 and 18 GGC repeats; thus the SBMA mutation has occurred at least twice, as previously indicated. $^{18}$

The Australian patients originated from two distinct areas, Sydney (AI1-AIX1) and Perth (AX1-AXV1), and two different founder haplotypes were found among them. The situation is, however, different from the Germans, since the two Australian founder haplotypes were carried equally by patients from both districts. All the Canadian patients carried different haplotypes, but two of them (CIV1 and CVIII1) harboured haplotypes similar to the two different Australian founder haplotypes. This is explained by the common immigration history of both these countries.
This, along with the fact that the two Australian haplotypes were not dependent on hospital districts, suggests that the mutations have occurred in these haplotypes in Europe before the ancestors of these patients emigrated to different continents. The British settlement of Australia did not start until 200 years ago. ${ }^{19}$ Also, the two Canadian patients with 'Australian' haplotypes, CIV1 and CVIII1, are British by origin. The ethnic diversity of the Canadian samples is clearly shown by the lack of a common Canadian haplotype. Hence, the SBMA mutation has occurred in different haplotypes among each original population.

The dependence between the CAG repeat length and the severity and age at onset of SBMA is controversial. In two studies $^{10,11}$ correlation between the repeat length and more severe symptoms and earlier onset of SBMA is reported, but in two other studies ${ }^{20,21}$ no correlation was found. In our study, weak negative correlation was found between the CAG repeat length and the age of onset in the 95 SBMA patients with defined ages at onset, but when the patients from different countries were examined separately, the correlation was detected only in the Japanese patients. The weakness of the correlation might be explained by the fact that our study did not include the largest extremes of repeats attainable in SBMA. The largest repeat included in the correlation dataset was $55 \mathrm{CAG}$ repeats $(\mathrm{JX})$, while repeats as long as 72 are reported in SBMA. ${ }^{7}$ Estimating the age of onset in SBMA patients is not simple. The patient primarily becomes conscious of muscular atrophy, but the first symptoms may have occurred 5-20 years before the patient consults a doctor because of muscular asthenia. Also, the progress of the disease is difficult to monitor because other symptoms caused by ageing intermingle with the symptoms of SBMA. There is no evidence of modifiers of clinical course in SBMA.

The fact that so many different founder haplotypes have thus far been identified in SBMA patients from different countries implies that the CAG repeat expansion mutation is not a unique event. No particular expansion-prone haplotype could be detected, in contrast to myotonic dystrophy (DM) (CTG repeat disease) and Friedreich's ataxia (GAA repeat disease), in which a predisposing haplotype associated with long normal repeats has been reported. ${ }^{13-15}$ Founder effects are also found in other CAG repeat diseases, eg SCA1 in Japan, ${ }^{22}$ SCA2 in Cuba, ${ }^{23}$ Japan $^{24}$ and India, ${ }^{25}$ MJD in Japan $^{26}$ and SCA6 in Germany. ${ }^{27}$ As these studies considered only restricted geographical areas, the presence of a predisposing haplotype is not clear. Besides, the SCA2 studies $^{23-25}$ revealed multiple founder haplotypes, indicating multiple origins for this disease. The CAG repeat in the AR gene is relatively stable, ${ }^{28}$ which explains the predominance of many country-specific founder haplotypes. Still, the SBMA CAG repeat seems to have expanded several times in different populations. 
14 Neville CE, Mahadevan MS, Barceló JM, Korneluk RG: High resolution genetic analysis suggests one ancestral predisposing haplotype for the origin of the myotonic dystrophy mutation. Hum Mol Genet 1994; 3: 45-51.

15 Cossée M, Schmitt M, Campuzano V et al: Evolution of the Friedreich's ataxia trinucleotide repeat expansion: Founder effect and premutations. Proc Natl Acad Sci USA 1997; 94: $7452-7457$

16 Ellegren $\mathrm{H}$ : Microsatellite mutations in the germline: implications for evolutionary inference. TIG 2000; 16: $551-558$.

17 Correa-Cerro L, Wöhr G, Häussler J et al: (CAG)nCAA and GGN repeats in the human androgen receptor gene are not associated with prostate cancer in a French-German population. Eur J Hum Genet 1999; 7: 357-362.

18 Tanaka F, Doyu M, Ito Y et al: Founder effect in spinal and bulbar muscular atrophy (SBMA). Hum Mol Genet 1996; 5: 1253-1257.

19 Cavalli-Sforza LL, Menozzi P, Piazza A: Australia, New Guinea and the Pacific Islands; in Cavalli-Sforza LL, Menozzi P \& Piazza A (eds) The History and Geography of Human Genes. Princeton University Press, Chichester, 1994, pp 343-371.

20 Amato AA, Prior TW, Barohn RJ, Snyder P, Papp A, Mendell JR: Kennedy's disease: a clinicopathologic correlation with mutations in the androgen receptor gene. Neurology 1993; 43: 791 794.

21 Morrison PJ, Mirakhur M, Patterson VH: Discordant repeat size and phenotype in Kennedy syndrome. Clin Genet 1998; 53: $276-277$

22 Wakisaka A, Sasaki H, Takada A et al: Spinocerebellar ataxia 1 (SCA1) in the Japanese in Hokkaido may derive from a single common ancestry. J Med Genet 1995; 32: 590-592.

23 Hernández A, Magarino C, Gispert S et al: Genetic mapping of the spinocerebellar ataxia 2 (SCA2) locus on chromosome 12q23-q24.1. Genomics 1995; 25: 433-435.

24 Mizushima K, Watanabe M, Kondo I et al: Analysis of spinocerebellar ataxia type 2 gene and haplotype analysis: (CCG)1-2 polymorphism and contribution to founder effect. J Med Genet 1999; 36: 112-114.

25 Saleem Q, Choudhry S, Mukerji M et al: Molecular analysis of autosomal dominant hereditary ataxias in the Indian population: high frequency of SCA2 and evidence for a common founder mutation. Hum Genet 2000; 106: 179-187.

26 Endo K, Sasaki H, Wakisaka A et al: Strong Linkage Disequilibrium and Haplotype Analysis in Japanese Pedigrees With Machado-Joseph Disease. Am J Med Genet 1996; 67: 437 - 444.

27 Dichgans M, Schöls L, Herzog J et al: Spinocerebellar ataxia type 6: Evidence for a strong founder effect among German families. Neurology 1999; 52: 849-851.

28 Watanabe M, Abe, K, Aoki M et al: Mitotic and meiotic stability of the CAG repeat in the X-linked spinal and bulbar muscular atrophy gene. Clin Genet 1996; 50: 133-137. 